\title{
COMPOSITION DES COMMISSIONS
}

\section{MEMBERSHIP OF COMMISSIONS}

$\dagger$ Members deceased since September 1967.

4. Commission des Ephémérides. (Ephemerides)

Président: G.A. Wilkins.

VICE-PRÉsIDENT: J. Kovalevsky.

Comité D'Organisation: G. M. Clemence, G. A. Čebotarev, R. L. Duncombe, W. Fricke.

Membres: Abalakin, Cunningham, de Greiff, Fayet, Gondolatsch, R. Haupt, Herrick, Lahiri, Lederle, Owaki, Rodriguez, Sadler (F. M. McBain), Sato, Sinzi, Trehan, Woolard.

Membres Consultants: H. C. Freiesleben.

5. Commission des Analyses de Travaux et de Bibliographie. (Abstracts and Bibliography)

(Committee of the Executive Committee)

Président: J. B. Sykes.

VICE-PRÉSIDENT: K.F. Ogorodnikov.

MemBres: Alter, Aoki, Beer, Belorizky, Bourgeois, Bouška, Chandrasekhar, Collinder, Dewhirst, Felber, Fricke, Harwood, Heinemann, Heintz, Henn, Hirose, Kleczek, Luplau-Janssen, Martynov, Pecker (J.-C.), Sticker, Wempe, Witkowski, Zinner.

Membres Consultants: A. Dunn, G. Feuillebois, A. Kemp, N. B. Lavrova, L. N. Radlova, I.S. Ščrbina-Samojlova.

6. Commission des Télégrammes Astronomiques. (Astronomical Telegrams)

(Committee of the Executive Committee)

Président : F. L. Whipple.

Vice-Président: P. Simon.

Membres: Buchar, Candy, Cunningham, Hers, Marsden, Martynov, Roemer.

7. Commission de la Mécanique Cẻleste. (Celestial Mechanics)

PRÉSIDENT: W.J. Eckert.

VICE-Président: G. N. Dubošin.

Comité D'Organisation: G.A. Čebotarev, Y. Hagihara, J. Kovalevsky, Y. Kozai, P.J. Message, K. Stumpff, V.G. Szebehely, F. Zagar.

Memrres: Aksenov, Barlier, Belorizky, Böhme, Bozis, Brumberg, Cesco, Clemence, Contopoulos, Cook (A.H.), Cox (J.F.), Cunningham, Danby, Deprit, Dubošin, Duncombe, Fabre, Ferraz-Mello, Galibina, Garfinkel, Giacaglia, Goldreich, Goudas, Hadjidemetriou, Hamid, Herget, Herrick, Hertz, Hori, Jeffreys, Katsis, Kaula, Kozai, Kustaanheimo, Marsden, Meffroy, Merman (G.A.), Morando, Musen, Popović, Rabe, Rjabov, Roy, Sadler (D. H.), Schubart, Sconzo, Sehnal, Sibahara, Siegel, Slavenas, Stiefel, Saraf, Thiry, Vinti, Wilkens, Wilkins.

Membres Consultants: V.I. Arnold, S. P. Diliberto, D. G. King-Hele, J. Moser, I.D. Žongolovič. 


\section{Commission de l'Astronomie de Position. (Positional Astronomy)}

Président: A. A. Nemiro.

Vice-Président: W. Fricke.

Comité D'Organisation: A. N. Adams, P. Lacroute, R.H. Stoy, R.H. Tucker, J.E.B. Von der Heide, H. Yasuda, M.S. Zverev.

Membres: Adams, Anguita, Argyrakos, Atkinson, Barros, Bohrmann, Dambara, Débarbat, Demetrescu, De Moraes, Dick, Dieckvoss, Dubois-Chevalier, Duncombe, Eichhorn, Fayet, Gliese, Gordon, Guinot, Haas, Hansson, Heintz, Hoffleit, Kosin, Larink, Laustsen, Lévy, Maître, Marcus, Melchior, Murray, Nefed'eva, Novopašennyj, Okuda, Petrov, Pilowski, Podobed, Položencev, Postoiev, Proverbio, Quijano, Reiz, Requième, Rybka (P.), Sandig, Scheepmaker, Schmeidler, Scott (F. P.), Sémirot, Slaucitajs, Symms, Ševarlić, Tavastšerna, Teleki, Thomas (D.V.), Tsubokawa, Tucker, Tuzi, Van Herk, Verbaandert, Wood (H.W.), Woolsey, Zimmermann.

\section{Commission des Instruments Astronomiques. (Astronomical Instruments)}

PRÉSIDENT: J. Rösch.

Vice-Président: V.B. Nikonov.

Comité D’Organisations: H.W. Babcock, W.A. Baum, R. G. Giovanelli, J.D. McGee, A. B. Meinel, O.A. Mel'nikov, B. Valníček.

MemBres: Aly, Atkinson, Bakos, Baranne, Barros, Beck, Behr, Bhattacharyya, Blitzstein, Brahde, Brealey, Brückner, Bowen (I.S.), Chincarini, Code, Couder, Cousins, Crawford, Delbouille, Delsemme, Dewhirst, Dimitroff, Dobronravin, Dollfus, Duchesne, Dunham, Ejnasto, Evans (J.W.), Fellgett, Ford, Gottlieb, Hall (J.S.), Høg, Hooghoudt, Ingrao, Ionnisiani, Jensch, Karandikar, Köhler, Kozyrev, Krasovskij, Kron (G.E.), Kurt, Lallemand, Linfoot, Linnik, Livingston, Meinel, Merman (N.V.) Mihel'son, Mohler, Monin, Moroz, Nikonov, Odgers, Petford, Pierce, Platzeck, Ramberg, Richardson (E. H.), Ring, Samaha, Scheffler, Seddon, Sisson, Smyth, Strand, Suharev, Sčeglov (V.P.), Väisälä, Wallenquist, Walker (M. F.), Walraven, Wayman, Wellgate, Wilcock, Wlérick, Zacharov. Membres Consultants: A. Bayle, L. E. Flory, Schwesinger, R. Wilson, C. J. Wynne.

\section{Commission de l'Activité Solaire. (Solar Activity)}

Président: Z. Svestka.

Vice-PrÉsIDENT: J.T. Jefferies.

Comité D'Organisation: K.O. Kiepenheuer, R. Michard, S. Nagasawa, G. Newkirk, J. Rösch, A.B. Severnyj, M. Waldmeier.

SECRÉTAIRE: A. D. Fokker.

MemBres: Allen, Aly, Athay, Babcock (H.W.), Balli, Banin, Bell (B.), Belorizky, Bhatnagar (A.), Billings, Bonov, Bray, Brückner, Bruzek, Bumba, Cardus, Cimino, Coutrez, De Feiter, De Groot, De Jager, Dezső, Dizer, Dodson-Prince, Dollfus, Dubov, Dunn, Elste, Ferraro, Fracastoro, Friedman, Fritzová-Švestková, Gaizauskas, Giovanelli, Gleissberg, Gnevyšev, Gnevyševa, Godoli, Goldberg, Gopasjuk, Gullón, Gurtovenko, Hanasz, Hedeman, Hirayama, Howard (R.F.), Hyder, Iošpa, Jäger, Jakimiec, Jakovkin (N.A.), Jensen, Kleczek, Kopecký, Krat, Křivský, Krüger (A.), Kuklin, Kundu, Künzel, Lehnert, Leighton, Leroy, Letfus, Loughhead, McKenna, Macris, Makita, Martres, Mathias, Mattig, Maxwell, Menzel, Mergentaler, Mitra, Mogilevskij, Mohler, Moiseev, Moreton, Mustel, Nishi, Notuki, Obridko, Öhman, Orrall, Pick-Gutmann, Popovici, Razmadze (T.S.), Richardson (R.S.), Ringnes, Roberts (W.O.), Rompolt, Saito (K.), Sakurai, Sarabhai, Schlüter, Schmidt (H.U.), Servajean, Shapley (A.H.), Sheeley, Simon (P.), Sitnik, Slonim, Smerd, Smith (E.v.P.), Smith (Henri J.), Stepanov, Stešenko, Sturrock, Suemoto, Swarup, Šapošnikova, Šklovskij, Tandberg-Hanssen, Trellis, Tuominen, Valníček, Van 't Veer (F.), Vitinskij, Von Klüber, Warwick (C.S.), Wild (J.P.), Wlérick, Xanthakis, Zirin, Zwaan. 
12. Commission de la Radiation et de la Structure de l'Atmosphère Solaire. (Radiation and Structure of the Solar Atmosphere)

Président: M.N. Gnevyšev.

VICE-PrésIDENT: R. G. Athay.

Comité D'Organisation: L. Delbouille, R. Michard, M. Minnaert, Miss Edith A. Müller, A.K. Pierce, Z. Suemoto.

Membres: Adam, Allen, Bappu, Billings, Blackwell, Blaha, Blamont, Böhm, Böhm-Vitense, Bray, Brück (H.A.), Bruzek, Christiansen, De Jager, Dubov, Edlén, Edmonds, Elste, Evans (J.W.), Gingerich, Giovanelli, Godoli, Gökdogan, Goldberg, Haupt (R. F.), Hotinli, Houtgast, Hubenet, Jäger, Jefferies, Kanno, Kato, Kawaguchi, Kiepenheuer, Konovovič, Kopecký, Laborde, Labs, Leighton, Leroy, Locke, Loughhead, Lüst (R.), Mathias, Matsushima, Mattig, Mergentaler, Migeotte, Moore-Sitterly, Mugglestone, Namba, Neven, Newkirk, Nicolet, Nishi, Orrall, Pande, Pajdušảková, Pecker (J.-C.), PeckerWimel, Peyturaux, Redman, Righini, Rigutti, Roland, Schröter, Seaton, Severnyj, Sitnik, Sobolev (V. M.), Swensson, Švestka, Tandberg-Hanssen, Thomas (R. N.), Tousey, Uchida, Unsöld, Voigt, Von Klüber, Waldmeier, Warwick (J.W.), Wilson (P.R.), Wlérick, Zirin, Zirker.

Membres Consultants: Mrs J.S. Duncombe.

14. Commission des Données Spectroscopiques Fondamentales. (Fundamental Spectroscopic Data)

Président: M. Migeotte.

Vice-Président: A.H. Cook.

Comité D'Organisation: R.H. Garstang, G. Herzberg, W. Lochte-Holtgreven, Mrs Ch. Moore-Sitterly, J.G. Phillips, R. Tousey.

Membres: Allen, Andrew, Baird, Bates, Blaha, Branscomb, Burgess, Carver, Douglas (A. E.), Dobronravin, Dressler, Dufay, Edlén, Engelhard, Essen, Felenbok, Garton, Green (L.C.), Herman, Hindmarsh, Humphreys, Jacquinot, Jordan (H.L.), Junkes, Kessler, Kiess $\dagger$, King (R. B.), Lawrence, Layzer, Littlefield, Lochte-Holtgreven, Martin Jr. (W. C.), Melnikov, Milford, Minnaert, Mohler, Monfils, Naqvi, Nevin, Nicholls, Obi, Peach, Prokof'ev (V.K.), Rigutti, Rosen, Salpeter (E.W.), Schadee, Seaton, Smit, Somerville, Swings, Takayanagi, Terrien, Traving, Trefftz, Van Bueren, Van Regemorter, Varsavsky, Wares, Wiese, Wilson (R.), Zirin.

Membres Consultants: W.S. Benedict, R.D. Cowan, H.M. Crosswhite, A. Dalgarno, H. Griem, V. Kaufman, A. Lagerquist, N. P. Penkin, I. Sobelman.

15. Commission pour l'Étude Physique des Comètes. (Physical Study of Comets)

PRÉSIDENT: L. Biermann.

ViCE-PRÉsident: V. Vanýsek.

Comité D'Organisation: G. Herzberg, B.J. Levin, E. Roemer, F. L. Whipple, K. Wurm.

Membres: Arpigny, Bertaud, Beyer, Bobrovnikoff, Bouška, Brown (P. Lancaster), Dobrovolskij, Dossin, Dufay (J.) †, Guiguay, Haser, Hunaerts, Liller (W.), Lüst (Rhea), Lyttleton, Martel-Chossat, Miller (F.D.), Pflug, Poloskov, Probstein, Rémy-Battiau, Richter (N.), Rijves, Sivaraman, Swings, Vsehsvjatskij, Waterfield.

16. Commission pour l'Étude Physique des Planètes et des Satellites. (Physical Study of Planets and Satellites)

PrÉSIDENT: J.S. Hall.

VICE-PRÉSIDENT: B. J. Levin.

Comité D'Organisation: A. Dollfus, G. Herzberg, G.P. Kuiper, C.H. Mayer, C.E. Sagan, R. Wildt.

Membres: Barabašov, Barreto, Baum, Bobrov, Brunk, Bullen, Camichel, Chamberlain, Collinson, Connes (J.), de Marcus, de Mottoni, de Vaucouleurs, Drake, Focas, Fox, 
Gehrels, Giclas, Goody, Guérin, Günther, Hunten, Ingrao, Irvine, Jeffreys, Jelley, Kaplan (L.), Kellog, Kiess †, Kopal, Koval, Levin, Liddell, Link, Lipskij, Luplau-Janssen, Martynov, Menzel, Middlehurst, Millman, Miyamoto, Narayana, O'Keefe, Öpik, Pettengill, Rösch, Runcorn, Safronov, Shoemaker, Sinton, Smith (B.A.), Smith (Harlan J.), Spinrad, Strong, Tombaugh, Whitaker, Wilson (A.G.).

Membres Consultants: R. Hide, G. Kennedy, G. McDonald, J. Mintz, J. B. Pollack.

17. Commission de la Lune. (The Moon)

Président: A. Dollfus.

VICE-Président : A. I. Lebedinskij $\uparrow$, G. H. Pettengill.

SECRÉTAIRE: B. M. Middlehurst.

Comité D'Organisation: G. P. Kuiper, D.H. Menzel, M.G. J. Minnaert.

Membres: Arthur, Ashbrook, Barabašov, Bell (B.), Boneff, Botelheiro, Cameron (W.), Drofa, Džapiašvili, Eckert, Elston, Ezerskij, Fielder, Focas, Gold, Green (J.), Guth, Habibullin, Hall (R.G.), Haupt (R.F.), Heyden, Hopmann, Ingrao, Jakovkin (A.A.), Jeffreys, Karandikar, Kopal, Levin, Liddell, Markowitz, Martynov, Matsushima, Millman, Moore (P.), Nefed'ev, Nicholson, O'Keefe, Potter, Rösch, Sadler (F. M. McBain), Sagan, Sato, Schrutka-Rechtenstamm, Shoemaker, Sinton, Strom (R.), Sytinskaja, Troickij, Ueta, Urey, Watts, Weimer, Whitaker.

19. Commission de la Rotation de la Terre. (Rotation of the Earth)

PrÉsident: P. J. Melchior.

Vice-Président: H. M. Smith.

Comité D'Organisation: E.P. Fedorov, B. Guinot, W. Markowitz, S. Yumi.

Membres: Abraham, Aksent'eva, Billaud, Bonanomi, Brkić, Buchar, Cecchini, Čudovičeva, Demetrescu, Dramba, Enslin, Essen, Fichera, Fleckenstein, Gama, Hall (R.G.), Hers, Iijima, Jeffreys (H.), Kalmykov, Koebcke, Kulikov, Lagrula, Lederle, Levallois, Meinig, Mihajlov, Miyadi, Narayana, Nicolini, Okuda, Opalski, Orte, Parijskij (N.N.), Pavlov, Popov, Postoiev, Proverbio, Randić, Rice, Romanskaja, Saharov, Scheepmaker, Sekiguchi, Stoyko (A.), Stoyko (N.), Sugawa, Ševarlić, Takagi, Tanner, Tardi, Teleki, Thomas (D.), Torao, Tsao, Vicente, Witkowski, Young (A.), Yumi.

20. Commission des Positions et des Mouvements des Petites Planètes, des Comètes et des Satellites. (Positions and Motions of Minor Planets, Comets and Satellites)

Président: G. A. Cebotarev.

VICE-PRÉSIDENT: F.K. Edmondson.

Comité D'Organisation: S. Arend, P. Herget, H. H. Hirose, H. W. Wood.

Memrres: Ahmed, Böhme, Boyer, Bruwer, Candy, Clemence, Cunningham, Debehogne, Evdokimov, Galibina, Gehrels, Giclas, Gutiérrez-Moreno, Herrick, Hertz, Itzigsohn, Jahontova, Kamieński, Kazimirčak-Polonskaja, Kovalevsky, Kresák, Kuiper, Makover, Marsden, Michkovitch, Milet, Missana, Orlov, Oterma, Popović, Porter, Protitch, Rabe, Rasmusen, Robertson, Roemer, Schmitt, Schrutka-Rechtenstamm, Schubart, Sekanina, Sitarski, Strobel, Torroja, Väisälä, Van Biesbroeck, Van Houten, Van Houten-Groeneveld, Wilkins, Zadunaisky.

21. Commission de la Luminescence du Ciel. (The Light of the Night Sky)

PRÉSIDENT: M. Huruhata.

Vice-Président: $H$. EIsässer.

Comité d'Organisation: J. Dufay $\dagger$, M.F. Ingham, A. Lebedinskij $\dagger$, F.E. Roach, G. Weill, J.L. Weinberg.

Membres: Asaad, Barber, Bates, Blackwell, Chamberlain, Chapman, Cook (A.F.), Dauvillier, Dufay (M.), Dumont, Elvey, Fesenkov, Fesenkova, Galperin, Gauzit, Grandmontagne, 
Harang, Haug, Hoffmeister $\uparrow$, Hunten, Jarrett, Kaplan (J.), Karandikar, Karjagina, Kastler, Krasovskij, Megrelišvili, Neužil, Nicolet, Pearse, Pettit-Knaflich, Robley, Sinvhal, Steiger, Tanabe, Vassy, Wallace, Weill, Weinberg.

Membres Consultants: Nguyen Doan.

22. Commission des Météores et Météorites. (Meteors and Meteorites)

PrésIDENT: Z. Ceplecha.

VICE-PRÉSIDENT: R. E. McCrosky.

Comité d'Organisation: P.B. Babadžanov, W.G. Elford, C.L. Hemenway, A.A. Javnel', P. M. Millman.

Membres: Abbott, Anders, Astapovič, Cook (A.F.), Davies, Davis (J.), Ellyett, Fedinskij, Fesenkov, Fireman, Gauzit, Guigay, Guth, Halliday, Hawkins, Hey, Hirose, Hodge, Hoffmeister $\dagger$, Hoppe, Jacchia, Kaiser, Katasev, Keay, Kizilirmak, Kostylev, Kresák, Kresáková, Krinov, Levin, Lindblad (B. A.), Lovell, McIntosh, Nazarova, Nielsen, O'Keefe, Oleak, Olivier, Öpik, Plavcová, Rajchl, Southworth, Verniani, Whipple.

Membres Consultants: Alexander, Henderson, Hey, Marvin, Mason, Sztroka y, Wickmann, Zahringer.

23. Commission de la Carte du Ciel. ('Carte du Ciel')

PrÉsident: W. Dieckvoss.

VICE-PRÉSIDENT: P. Lacroute.

Comité D'Organisation: P. Couderc, A. N. Dejč, P. Herget, H. Kox, W. J. Luyten, P. Sémirot, H.W. Wood.

Membres: Abhyankar, Bouigue, Dessy, Eichhorn, Fracastoro, Gallouët, Günther, Harris, Lourens, Murray, O'Connell.

24. Commission des Parallaxes Stellaires et des Mouvements Propres. (Stellar Parallaxes and Proper Motions)

Président: W.J. Luyten.

VICE-PRÉsIDENT: S. Vasilevskis.

Comité d'Organisation: A.N. Dejč, V.V. Lavdovskij, J.v. B. Lourens, W.W. Morgan, C.A. Murray, K. Aa. Strand, P. van de Kamp.

Membres: Blaauw, Cecchini, Delhaye, Dieckvoss, Eichhorn, Fatčihin, Franz, Fredrick, Giclas, Gliese, Goyal, Haas, Harris, Hoffleit, Kohlschütter, König, Lippincott, Meurers, Missana, Moreno, Paloque, Schilt, Smart, Stearns, Vyssotsky, Wagman, Wilkens.

Membres Consultants: W.F. Van Altena.

25. Commission de Photométrie Stellaire. (Stellar Photometry)

Président: A.W.J. Cousins.

VICE-PRÉsIDENT: D. L. Crawford.

Comité d’Organisation: A.N. Argue, M. Golay, R.H. Hardie, G.E. Kron, V.B. Nikonov, E. Rybka, J. Stock.

MemBres: Bahng, Becker (W.), Behr, Bigay, Bok (B.J.), Bok (P.F.), Borgman, Brück (H.A.), Chis, Dufay (J.) †, Eelsalu, Elvius (T.), Feinstein, Fellgett, Fitch, Gallouët, Graham, Gutiérrez-Moreno, Haffner, Hertzsprung $\dagger$, Hiltner, Hoag, Holmberg, Irwin, Jackisch, Johnson (H.L.), McCarthy, Masani, Mayer (P.), Mianes, Moreno, Muller (A.B.), Mumford, Notni, Osawa, Philip, Quijano, Schmidt (H.), Smyth, Steinlin, Strajžic, Strohmeier, Velghe, Wallenquist, Walraven, Wesselink, Willstrop, Young (A.T.).

26. Commission des Étoiles Doubles. (Double Stars)

Président: P.C. Couteau.

VICE-PrésIDENT: J. Dommanget. 
Comité D’Organisation: O.J. Eggen, P. Kulikovskij, P. Muller, K.Aa. Strand, P. van de Kamp.

Membres: Arend, Baize, Batten, Cester, Dejč (A.N.), Dhonomidjojo, Dick, Djurković, Finsen, Franz, Güntzel-Lingner, Hadjidemetriou, Heintz, Hertzsprung †, Holden, Hopman, Jeffers, Johnson (M.C.), Jonckheere, Kuiper, Kumar, Lippincott, Luplau-Janssen, Luyten, Mourao, Van Albada, Van Biesbroeck, Van den Bos, Wierzbinski, Wieth-Knudsen, Worley, Zagar.

27. Commission des Étoiles Variables. (Variable Stars)

Président: L. Detre.

Vice-Président: O.J. Eggen.

Comité d'Organisation: A. A. Bojarčuk, G. H. Herbig, R. Kippenhahn, K. Kwee, G. Odgers.

Membres: Ahnert, Alanija, Arp, Ashbrook, Balázs-Detre, Banerji, Bateson, Bertaud, Bhatnagar (P.L.), Cesevič, Chavira, Chis, Christy, Daube-Kurzemniece, de Kock, Deutsch (A.J.), Efremov, Eskioglu, Feast, Fernie, Fitch, Gaposchkin, Geyer, Haro, Harwood, Hertzsprung †, Heyden, Hoffleit, Hoffmeister †, Holopov, Huffer, Huruhata, Ikaunieks, Išcenko, Jarzebowski, Jones, Kopylov, Kordylewski, Kraft, Kukarkin, Kumsišvili, Lacchini, Ledoux, Lortet-Zuckermann, McNamara, Mannino, Masani, Mavridis, Mayall (M.W.), Miller (W.J.), Nielsen, O'Connell, Oosterhoff, Opolski, Oskanjan, PayneGaposchkin, Peltier, Piotrowski, Plaut, Plavec, Rakos, Richter (G.), Rodgers, Romano, Rosino, Sawyer-Hogg, Shapley (H.), Slavenas, Smak, Smith (Harlan J.), Strohmeier, Swope, Tempesti, Vandekerkhove, Van Hoof, Wachmann, Walker (M.F.), Walraven, Wenzel, Wesselink, Wright (F.W.), Xanthakis, Zinner, Zwicky.

28. Commission des Galaxies. (Galaxies)

Président: G.C. McVittie.

Vice-Président: E. M. Burbidge (Mrs).

SeCrÉTAIRE: E. Holmberg

Comité D’Organisation: D.S. Evans, D. Heeschen, E. Holmberg, B.E. Markarjan, R.L. Minkowski, M. Schmidt.

Membres: Abell, Alfvén, Ambarcumjan, Arp, Baum, Bertola, Bigay, Boischot, Bok, Bondi, Bonnor, Burbidge (G.R.), Contopoulos, Courtès, Dessy, de Vaucouleurs, Elvius (A.M.), Field, Gascoigne, Haro, Heckmann, Heidmann, Hindman, Hodge, Hoyle, Humason, Johnson (H.M.), Kalinkov, King (I.R.), Kundu, Kustaanheimo, Layzer, Lindblad (P.O.), McCrea, Madwar, Mayall (N.U.), Mills, Morgan, Narlikar, Neyman, Oort, Omer, Osterbrock, Pachner, Page, Pishmish de Recillas, Poveda, Prendergast, Reaves, Reddish, Richter (N.B.), Rindler, Roberts (M.S.), Robinson (I.), Rosino, Rubin, Rudnicki, Sandage, Schmidt (M.), Schücking, Schürer, Sciama, Scott (E.L.), Serrsic, Shakeshaft, Shane (C.D.), Shapley (H.), Spinrad, Stibbs, Thackeray, Treder, Vandekerkhove, Van den Bergh, Voroncov-Vel'jaminov, Westerlund, Whitford, Whitrow, Wild (P.), Wilson (A.G.), Zel'dovič, Zwicky.

29. Commission des Spectres Stellaires. (Stellar Spectra)

PRÉSIDENT: M.W. Feast.

VICE-PRESIDENT: Y. Fujita.

Comité d’Organisation: M.K.V. Bappu, G. Herbig, L. Houziaux, I. M. Kopylov, A. Przybylski, J. Sahade.

Membres: Abhyankar, Abt, Aller, Andrillat (H.), Andrillat (Y.), Asaad, Babcock (H.W.), Berger, Bertaud, Bidelman, Bloch, Boggess, Bojarčuk, Bouigue, Bretz, Brück (H.A.), Buscombe, Cayrel-de-Strobel (G.), Cayrel (R.), Chalonge, Code, Conti, de Groot, Deutsch (A.J.), Divan, Dobronravin, Dolidze, Dunham, Edmonds Jr., Evans (D.S.), Fringant, Gollnow, Gorbackij, Gratton, Greenstein, Griffin (R.F.), Groth, Guthrie, Hack, 
Haro, Heard, Heintze, Henize, Herman, Huang, Iwanowska, Jaschek (C.O. R.), Jaschek (M.), Jugaku, Keenab, Kienle, King (R.B.), Kraft, Larsson-Leander, McCuskey, McNamara, Mannino, Mel'nikov, Milligan, Morton (D.C.), Mustel, Nicholls, Nikitin, Oetken, Oke, Osawa, Payne-Gaposchkin, Pedoussaut, Ringuelet-Kaswalder, Rosen, Sargent, Searle, Slettebak, Smak, Stawikowski, Stecher, Svolopoulos, Swensson, Swings, Taffara, Thackeray, Thompson (G.I.), Underhill, Vandekerkhove, Van 't Veer-Menneret, Voigt, Wallerstein, Wehlau, Wellmann, Wempe, Willstrop, Wilson (O.C.), Wilson (R.), Wright (K.O.), Wyller, Yamashita.

30. Commission de Vitesses Radiales. (Radial Velocities)

Président: D.S. Evans.

Vice-Président: H.A. Abt

SeCrétaire: W. Buscombe.

Comité D'Organisation: A. H. Batten, A. Blaauw, R. Bouigue, A. D. Thackeray.

Membres: Abt, Beardsley, Bertiau, Boulon, de Vaucouleurs, Edmondson, Fehrenbach, Gollnow, Gratton, Griffin (R.F.), Harding, Heard, Kraft, Northcott, Oetken, Pagel, Preston, Rubin, Sahade, Underhill, Wayman.

31. Commission de l'Heure. (Time)

PRÉSIDENT: F. Zagar,

Vice-Président: G.M.R. Winkler

Comité D'Organisation: D.J. Belocerkovskij, J. Bonanomi, H. Enslin, W. Markowitz, H.M. Smith, M. Torao.

Membres: Abraham, Arbey, Bakulin, Billaud, Blaser, Brkić, Decaux, Demetrescu, Dingle Dramba, Dubois-Chevallier, Fuchs, Gama, Gökmen, Gougenheim, Guinot, Hall (R.G.), Hemmleb, Hers, Iijima, Koebcke, Lacombe, Lederle, Lorón, Madwar, Melchior, Mihailov, Miyadi, Opalski, Orte Lledo, Pavlov, Postoiev, Proverbio, Randić, Sandig, Stoyko (A.), Stoyko (N.), Ščeglov, Širjaev, Šternberk, Takagi, Tardi, Thomson (M. M.), Tsao, Verbaandert, Von der Heide, Winkler.

Membres Consultants: U. Adelsberger, L. Essen, J. Terrien.

33. Commission de la Structure et de la Dynamique du Système Galactique. (The Structure and Dynamics of the Galactic System)

Président: G. Contopoulos.

Vice-Président: S. W. McCuskey.

Comité d'Or ganisation: A. Blaauw, B.J. Bok, E. K. Haradze, F.J. Kerr, G.G. Kuzmin, C.C. Lin, M. Schmidt.

MemBres: Aarseth, Agekjan, Ambarcumjan, Baldwin, Bidelman, Bigay, Barbanis, Becker (W.), Beer, Blanco, Boulon, Burke, Crawford, Delhaye, Dieter, Dzigvašvili, Edmondson, Ejnasto, Elsässer, Elvius (A.M.), Elvius (T.), Fehrenbach, Fenkart, Fricke, Goldreich, Gyldenkerne, Haug, Heckmann, Hénon, Holopov, Hunter (C.), Idlis, Ikaunieks, Irwin, Iwaniszewska-Lubienska, Jaschek (C.O.R.), Johnson (H.M.), Kaburaki, King (I.R.), Kinman, Kukarkin, Kurth, Lecar, Lindblad (P.O.), Lunel, Luyten, Lynden-Bell, McCarthy, McRae, Malmquist, Mavridis, Mirzojan, Mohr, Morgan, Münch (G.), Murray, Nahon, Ogorodnikov, Ollongren, Oort, Ovenden, Perek, Perry, Pesch, Philip, Pilowski, Priester, Ramberg, Roman, Ruben, Rudnicki, Schalén, Schilt, Schmidt (H.), Schmidt (K.H.), Schmidt-Kaler, Sarov, Shimizu, Slettebak, Steinlin, Stibbs, Szebehely, The (Pik Sin), Toomré, Upgren, Vanderlinden, Vandervoort, Van Hoof, Varsavsky, Velghe, Vetešnik, Wayman, Weaver, Westerhout, Westerlund, Woltjer, Woolley.

34. Commission de la Matière Interstellaire et des Nébuleuses Planétaires. (Interstellar Matter and Planetary Nebulae)

PrésIDENT: D. E. Osterbrock. 
VICE-PrÉSIDENT: F. D. Kahn.

Comité D'Organisation: L. H. Aller, M. J. Greenberg, S. B. Pikel'ner, L. Rosino.

Membres: Andrillat (H.), Andrillat (Y.), Baldwyn, Behr, Bok (B.J.), Chopinet, Chvojková, Courtès, Davies (R.D.), Dieter, Dibaj, Divan, Dunham, Friedemann, Elvius (A.M.), Gehrels, Goldsworthy, Grzedzielski, Gurzadjan, Hall (J.S.), Haradze, Havtasi, Henize, Hiltner, Herbig, Johnson (H.M.), Kaplan (S.A.), Kerr, Kipper, Kogure, Kohoutek, Lambrecht, Liller, Loden, McCrea, McNally, Martel-Chossat, Menon, Minin, Minkowski, Münch (G.), Nandy, Osaki, Ozernoj, Pottasch, Razmadze (N. A.), Rožkovskij, Safronov, Savedoff, Schalén, Schatzman, Schmidt (K.H.), Schmidt-Kaler, Seaton, Seddon, Serkowski, Sharpless, Šklovskij, Sobolev, Spitzer, Strömgren, Syrovatskij, Takakubo, Thackeray, Thompson, Treanor, Van de Hulst, Van Woerden, Vanýsek, Viswanathan, von Hoerner, Voroncov-Vel'jaminov, Walker (G.A.H.), Westerhout, Whitford, Wickramasinghe, Zimmermann (H.).

Membres Consultants: S. A. Colgate

35. Commission de la Constitution des Étoiles. (Stellar Constitution)

Président: A. G. Masevič (Mrs).

Vice-Président: E. Schatzman.

Membres: Bhatnagar (L.), Biermann, Bondi, Boury, Brownlee, Burbidge (G.R.), Cameron (A.G.W.), Carson, Chandrasekhar, Chiu, Christy, Cowling, Cox (A.N.), Cox (J.P.), Demarque, Dingens, Epstein (J.), Ezer, Ferraro, Fowler, Gamow, Gjellestad, Hayashi, Henyey, Hitotuyanagi, Hoyle, Kaplan (S.A.), Kippenhahn, Kothari, Krook, Kumar, Kushwaha, Lebedinskij $\dagger$, Lebovitz, Ledoux, McCrea, Marx, Masani, Mestel, Reeves (H.), Reiz, Rosseland, Roxburgh, Ruben, Salpeter (E.E.), Savedoff, Schwarzschild, Sears, Spiegel, Strömgren, Suda, Sweet, Tayler, Temesváry, Tuominen, Van der Borght, Vardya, Weigert, Wrubel, Ževakin.

36. Commission de la Théorie des Atmosphères Stellaires. (The Theory of Stellar Atmospheres)

PRÉSIDENT: A. B. Underhill (Miss).

Vice-Président: R. Cayrel.

Comité D'Organisation: K. H. Böhm, V. V. Ivanov, J. T. Jefferies, R. N. Thomas, S. Ueno.

MemBres: Abhyankar, Aller (L.H.), Athay, Avrett, Baschek, Biermann, Böhm-Vitense, Busbridge, Carson, Cayrel (G.), de Feiter, Edmonds, Elste, Gebbie, Gingerich, Godoli, Gökdogan, Grant, Greenstein, Groth, Gussmann, Hack, Hardorp, Hearn, Henyey, Hitotuyanagi, Hotinli, Houtgast, Houziaux, Huang, Hummer, Hunger, Johnson (H. R.), Kalkofen, Kumar, Krishna Swamy, Kushwaha, McDonald (J.K. Petrie), Matsushima, Menzel, Mihalas, Mirzojan, Miyamoto, Müller (E. A.), Münch (G.), Mustel, Neven, Orrall, Pagel, Pecker (J.-C.), Pecker-Wimel, Petrie (J.K. McD.), Phillips, Pottasch, Rudkjøbing, Saito (S.), Schatzman, Sitnik, Sobolev, Sobouti, Spiegel, Stibbs, Strom (S.), Swihart, Traving, Uesugi, Unno, Unsöld, Van Regemorter, Van 't Veer (F.), Vardya, Weidemann, Wellmann, Whitney (C.A.), Wright (K.O.), Wrubel, Wyller, Zirker, Zwaan.

37. Commission des Amas Stellaires et des Associations. (Star Clusters and Associations)

Président: M. Golay.

VICE-PRÉsident: G. Larsson-Leander.

Comité d’Organisation: Mrs K.A. Barhatova, S.B.C. Gascoigne, I.R. King, L. Rosino, J. Ruprecht, Miss H. H. Swope, M.F. Walker.

Membres: Aarseth, Alter, Artjuhina, Balázs, Becker (W.), Blaauw, Bouvier, Cuffey, Eggen, Feast, Feinstein, Golay, Haffner, Hénon, Holopov, Hoag, Johnson (H. L.), Kadla, Lavdovskij, Lodén (K.), Lohmann, Lynden-Bell, Mavridis, Menon, Meurers, Morgan, Pishmish de Recillas, Reddish, Šarov, Sandage, Sawyer-Hogg, Serkowski, Shapley (H.), Smyth, Takase, The, Van den Bergh, Vanderlinden, Van Schewik, von Hoerner, Walker (G.A.H.), Wallenquist, Weaver, Woolley. 
38. Commission pour l'Échange des Astronomes. (Exchange of Astronomers)

(Committee of the Executive Committee)

Président: M.G. J. Minnaert

Vice-Président: A. Reiz

Comité d’Organisation: D. Ja. Martynov, P. M. Routly, J. Sahade, J.P. Wild.

Membres: Abetti, Babcock (H.D.) ${ }^{\dagger}$, Bappu, Bok, Bowen, Delhaye, Keller, Kienle, Kourganoff, Mihajlov, Miyamoto, Mohr, Page, Righini, Rosseland, Stoy, Swings, Witkowski, Wood (F. B.), Wyatt.

Membres Consultants: E. M. Fournier d'Albe

40. Commission de la Radioastronomie. (Radio Astronomy)

Président: J.P. Wild.

Vice-Président: D.S. Heeschen. $\because$,

Comité D’Organisation: N. Kardašev, M. R. Kundu, J. Lequeux, M. Ryle, S.F. Smerd, G. Westerhout.

Membres: Abrami, Akabane, Atanasijević, Avignon, Baldwin, Baltá-Elias, Barret, Blum, Boischot, Bolton, Bracewell, Brown (R. Hanbury), Burbidge (G.R.), Cardus, Carr, Christiansen, Chvojková, Cohen, Costain, Coutrez, Daene, Davies (J.G.), Davies (R.D.), de Groot, Denisse, Dewhirst, Dieter, Dodson-Prince, Drake, Elwert, Eriksen, Field, Findlay, Firor, Fokker, Friedman, Galt, Ginzburg, Gold, Gorgolowski, Grahl, Hachenberg, Haddock, Hagen, Hajkin, Harang, Harrower, Heidmann, Hewish, Hey, Hill (E. R.), Howard (W.E.), Hughes (J.A.), Ihsanova, Kahn, Kakinuma, Kazès, Kerr, Kraus, Laffineur, Le Squeren, Letfus, Lilley, Little (A.G.), Locke, Lovell, McClain, McGee (R.X.), McKinley, MacRae, Macris, McVittie, Mannino, Maxwell, Mayer (C.H.), Menon, Mills, Minkowski, Moriyama, Muller (C. A.), Oort, Osterbrock, Parijskij (Ju. N.), Pick-Gutmann, Priester, Rabben, Righini, Roberts (J.A.), Rydbeck, Sanamjan, Scheuer, Schmidt (M.), Scott (P.F.), Seeger, Shakeshaft, Šklovskij, Simon (P.), Slee, Smith (A.G.), Smith (F.G.), Smith (Harlan J.), Soboleva, Stahr-Carpenter, Steinberg, Swarup, Takakura, Thompson (A. R.), Tlamicha, Townes, Troickij, Van Bueren, Van de Hulst, Van Woerden, Varsavsky, Vitkevič, Wade, Walsh, Weaver, Yen, Železnjakov.

41. Commission de l'Histoire de l'Astronomie. (History of Astronomy)

Président: E. Rybka.

VICE-PRÉsIDENT: O. Gingerich.

Comité D'Organisation: J.O. Fleckenstein, C.D. Hellman, Z. Horský, P.G. Kulikovskij, W. Petri, V.L. Cenakal.

Membres: Abetti, Argyrakos, Ashbrook, Baehr, Beer, Birkenmajer $\dagger$, Collinder, Dick, Dingle, Dobrzycki, Douglas (A.V.), Erpylev, Evans (D.S.), Felber, Ferrari d'Ochieppo, Filliozat, Hirose, Kamienski, King (H.C.), Kotsakis, Lévy, Link, Michel, Michkovitch, Nielsen, Norlind, Nørlund, Omer, Ovenden, Pelseneer, Pogo, Samaha, Slavenas, Sticker, Whitrow, Yabuuti, Zagar, Zinner.

Membres Consultants: Aaboe, Burke-Gaffney, Daumas, Fischer, Forbes, Freiesleben, Hartner, Horský, Hoskin, Kennedy, Labat, Maddison, Millas-Vallicrosa, Musto, Needham, Nevskaja, Pedersen, Price, Przypkowski, Ronan, Ronchi, Santillana, Schove, Taton, Thoren, Tumanjan, Veselovsky, van den Waerden, Wattenberg, Woolf, Wright.

42. Commission des Étoiles Doubles Photométriques. (Photometric Double Stars)

Président: F. B. Wood. I " " *

Vice-Président: M. Plavec.

Comité D'Organisation: A.H. Batten, T. Herczeg, M. Kitamura, G. Larsson-Leander, J.E. Merrill, V.P. Cesevič.

Membres: Abhyankar, Bappu, Beer, Binnendijk, Blitzstein, Broglia, Cester, Chis, Cillié, 
Cousins, Dadaev, de Kort, Ebbighausen, Ferrari d'Occhieppo, Fracastoro, Fredrick, Fresa, Gaposchkin (S.), Grygar, Günther, Gyldenkerne, Hardie, Heard, Huang, Huffer, Irwin, Kizilirmak, Koch, Kopal, Knipe, Krat, Kristenson, Kron (K.G.), Kruszewski, Kwee, Landolt, Lavrov, Linnell, Magalašvili, Martynov, Mumford, Nelson, O’Connell, Ovenden, Piotrowski, Plaut, Popovici, Popper, Rakos, Sahade, Schmidt (H.), Serkowski, Shapley (M. B), Shulberg, Sinvhal, Strohmeier, Szafraniec, Van Hoof, Walter, Wellmann, Wesselink, Whitney (B.S.), Widorn, Wright (K.O.).

43. Commission des Plasmas et Magnéto-Hydrodynamique en Astrophysique. (Astrophysical Plasmas and Magnetohydrodynamics)

PRÉSIDENT: L. Davis Jr.

Vice-Président: R. Lüst.

Comité D’Organisation: T. G. Cowling, S.A. Kaplan, B. Lehner.

Membres: Alfvén, Axford, Banerji, Bel, Bhatnagar (P.L.), Bierman, Bumba, Chandrasekhar, Csada, Ferraro, Ginzburg, Jensen, Kopecký, Krause, Kushwaha, Ledoux, Mestel, Parker, Piddington, Pikel'ner, Schlüter, Severnyj, Simon (R.), Smerd, Spitzer, Sturrock, Sweet, Šklovskij, Talwar, Tayler, Trehan, Van Bueren, Wentzel.

Membres Consultants: C. P. Sonett, S. N. Vernov.

44. Commission des Observations Astronomiques au-dehors de l'Atmosphère Terrestre. (Astronomical Observations from outside the Terrestrial Atmosphere)

Président: R. Wilson.

Vice-Président: V.K. Prokof'ev.

Comité D'Organisation: J.E. Blamont, C. de Jager, Goldberg, R. Lüst, F.G. Smith, R. Tousey.

Membres: Alexander (J.K.), Bogess, Boneff, Bosman-Crespin, Boyd, Brandt (J.C.), Butler, Carver, Chamberlain, Chubb, Code, Coutrez, Danielson, Deutsch (A.J.), Fazio, Fisher (P.C.), Fredga, Friedman, Giacconi, Ginzburg, Gold, Gratton, Haddock, Hallam, Hartz, Heddle, Henize, Herzberg (G.), Hinteregger, Houziaux, Huguenin, IvanovHolodnuj, Kraushaar, Kuiper, Kupperian, Lindblad (B. A.), Lines, Link, Lovell, McGee (J.D.), Mandel'stam, Masevič, Mehltretter, Meinel, Migeotte, Mihailov, Milford, Monfils, Morton (D.C.), Müller (Edith A.), Neupert, Newkirk, Nicolet, Nikolsky, Öhman, Parkinson, Pecker (J.-C.), Pounds, Priester, Reeves (E. M.), Rense, Righini, Rogerson, Roman, Rosen, Sarabhai, Schwarzschild, Smith (Henry J.), Steinberg, Underwood, Van de Hulst, Walsh, Whipple, Wilson (A. G.), Zwicky, Žongolovič.

Membres Consultants: M. Florkin, H. Glasser, E. A. Lauter, G. Occhialini, B.F. de Veubeke.

45. Commission des Classifications Spectrales et Indices de Couleur à Plusieurs Bandes. (Spectral Classifications and Multi-band Colour Indices)

Président: Ch. Fehrenbach.

VICE-Président: B. Westerlund.

Comité D'Organisation: W.P. Bidelman, E.K. Haradze, C.O.R. Jaschek, H.L. Johnson, W.W. Morgan.

Membres: Bahng, Barbier-Brossat, Bartaja, Beer, Blanco, Borgman, Boulon, Boyce, Buscombe, Cameron (R.C.), Canavaggia, Cester, Chalonge, Cowley, Dessy, Divan, Duflot (M.), Elvius (T.), Evans (D.S.), Feast, Golay, Griffin (R.F.), Gyldenkerne, Hack, Hallam, Henize, Herman, Hiltner, Hoag, Iwanowska, Jaschek (M.), Keenan, Kron (G. E.), Lodén (L.O.), Lodén (K.), Low, McCarthy, McCuskey, McNamara, Malmquist, Meinel, Mendoza, Morgan, Nikonov, Notni, Oosterhoff, Osawa, Perry, Philip, Preston, Reddish, Redman, Roman, Sanwal, Schmidt-Kaler, Seitter, Sharpless, Sinnerstad, Sinvhal, Slettebak, Steinlin, Stephenson, Strajžis, Strömgren, Thackeray, The, Treanor, Van den Bergh, Velghe, Walker (G.A.H.), Warner, Williams (J.A.), Wilson (O.C.), Yoss. 
46. Commission pour l'Enseignement de l'Astronomie. (The Teaching of Astronomy)

Président: E. A. Müller (Miss).

VICE-PRÉSIDENT: J. Kleczek.

Comité D'Organisation: G.O. Abell, E.K. Haradze, E. V. Kononovič, V. Kourganoff, M. G. J. Minnaert, T. L. Page, E. Schatzman, T. L. Swihart.

Membres: Dinulescu, Lambrecht, Rodgers. 